\title{
Los espejos de la infancia: retratos y autorretratos de niños/as en situación de calle
}

\section{Introducción}

Este trabajo surge de la investigación producida para la realización de mi tesina de grado de la Licenciatura en Trabajo Social, a partir de mi inclusión, en el mes de diciembre de 2006, en el Programa de Promoción Familiar del Área Niñez de la Municipalidad de Rosario como operadora de calle, en la zona norte de la ciudad.

A partir de los registros de campo entonces elaborados (con la intención paralela de su utilización para dicha investigación) se han captado modos de ser, hacer y decir de estos niños/as que, articulados con sucesos vivenciados in situ, junto a ellos, en el ámbito mismo de la calle, fueron resignificados a partir de su relectura y agrupación en ejes de interés para el análisis y la reflexión teórica. Los mismos intentan dar cuenta de la representación de los niños/as acerca de la calle y del concepto de trabajo, de la distribución de actividades en su vida cotidiana y del lugar o no de lo lúdico/recreativo en sus actividades en calle.

Se pretendió indagar así en la autorrepresentación que tienen acerca de su situación, los niños/as que realizan actividades laborales callejeras informales en la zona norte de la ciudad de Rosario, entendiendo a la autorrepresentación, tal como afirma Moscovici (1981), como la manifestación concreta de una toma de posturas sobre la identidad y la autopercepción y el modo en que ambas se conjugan para manifestarse de forma pública, para los Otros, y se ubican como detonador de un «estoy aquí».

Se intentan recuperar en este proceso, las pertenencias de las que se apropian y las relaciones que se van construyendo en su vida cotidiana, a partir de lo que se visibiliza en el espacio de la calle.

\section{Consideraciones metodológicas}

La posibilidad de basarse en registros realizados en el momento mismo en que las situaciones y los relatos tienen lugar, o en los instantes próximamente posteriores, otorga otras características a la investigación, permitiendo recuperar la riqueza de ciertos detalles en el ámbito callejero mismo. El trabajo de campo posibilita, asimismo, un ida y vuelta permanente desde la reflexión hacia el objeto empírico y desde éste nuevamente hacia el análisis. 
El tipo de registro etnográfico, originario de la antropología, aporta una perspectiva rica al Trabajo Social: «la flexibilidad teórica, desplazando la tradición de dividir el trabajo de campo con el momento analítico» (Moreta et alli, 2004: 5), y a la vez el Trabajo Social otorga otra mirada proveniente directa o indirectamente de la formación profesional.

El acercamiento logrado a partir del trabajo en terreno permite dar cuenta de una realidad específica, en un contexto geográfico, cultural, social e histórico determinado y queda plasmado en el registro de campo que, tal como explicita Guber (1990), se constituye como una herramienta de recolección de información en la cual se inscribe la interacción con los sujetos mediante diferentes vías de acceso (observación, charlas informales, experiencias vivenciales).

En el caso de la investigación mencionada, los registros fueron realizados en diferentes días de la semana (días hábiles) y en distintos horarios del día (mayoritariamente por la mañana, de 10 a 12 horas, y por la tarde, de 16 a 19 horas). Vale aclarar que son los momentos de mayor permanencia de niños/as en situación de calle, los que marcan la organización de los horarios de trabajo.

El recorte temporal utilizado está marcado por el semestre que va desde enero a junio de 2007, por considerar este lapso un tiempo considerable para caracterizar la población de la zona teniendo en cuenta los cambios estacionales, el comienzo del año lectivo, entre otras circunstancias, que pueden marcar variaciones cuantitativas y cualitativas de los niños/as en calle. Se efectuaron en estos meses cerca de cien registros de situaciones, en las cuales el acercamiento con los niños/as varía en intensidad y duración ${ }^{1}$.

Para la obtención de los registros fue esencial la utilización de la técnica de observación participante, técnica por la cual, tal como afirma Maestre (1990), se intenta captar la realidad social y cultural de una sociedad o grupo determinado, mediante la inclusión del investigador en el colectivo objeto de su estudio. En este caso, realizada desde una posición particular, la cual Amezcúa (2000) denomina la del «participante observador», en tanto tuvo lugar en escenarios con participación profesional del investigador.

El trabajo etnográfico se realiza en un determinado campo o emplazamiento. En este caso, el mismo está dado por el ámbito de la calle, en una zona geográfica determinada, con niños/as que transcurren parte de la jornada allí y van moviéndose dentro de ciertos límites, de acuerdo a la variedad en la localización de los sujetos. En este sentido, es importante marcar que el trabajo en calle tiene su particularidad en el hecho de desarrollarse en un ámbito que, al ser espacio de tránsito, pasa a constituirse para los niños/as y, en consecuencia, para el operador como espacio de permanencia.

Allí el trabajo tiene características peculiares, en tanto las actividades diversas desarrolladas por los niños/as marcan la necesidad de una adaptación permanente del operador en función de los tiempos posibles para el acercamiento y el diálogo. Es en esta realidad, marcada por el movimiento permanente, por la transitoriedad, donde es preciso fomentar un espacio que permita la formación de un vínculo. Vínculo que sólo es posible a partir del establecimiento de una relación dialógica que permita desplegar la subjetividad. Al mismo tiempo, la intención se centra en generar una cadena de acciones para el me-

${ }^{1}$ Muchos de los registros figuran de forma textual en la tesina y fueron suprimidos para el presente artículo, a los fines de no excedernos en la extensión. 
joramiento de la calidad de vida de los involucrados, el aporte en la resolución de situaciones problemáticas y la problematización de otras que se encuentran naturalizadas.

Fue la relación basada en el diálogo y sin la estructuración que podría marcar, por ejemplo, una serie de preguntas pautadas, a modo de entrevista, la que facilitó el desarrollo de esta investigación desde la perspectiva etnográfica, superando el «relevamiento de datos» e incluyendo para el análisis la perspectiva de contexto.

El desarrollo del trabajo se produjo en el denominado «circuito Norte»², zona al interior de este distrito delimitada a partir de los lugares donde se encuentran niños/as en situación de calle. Estos puntos coinciden con lugares de gran tránsito de personas y vehículos y una importante zona comercial y de servicios.

No es un dato menor destacar que la población a la cual se hace referencia es casi exclusivamente de género masculino, habiéndose registrado escasas situaciones de niñas en el circuito, generalmente acompañadas de hermanos varones, o bien de familiares adultos.

En relación a las edades, las franjas etarias en las cuales es mayor la cantidad de niños/as en situación de calle en el período que toma el estudio son las de siete a nueve años de edad (acompañados por algún hermano de la misma edad o algunos años mayor) y adolescentes de dieciséis a dieciocho años (que se caracterizan por encontrarse mayoritariamente en grupos). La franja que va de los trece a los quince años de edad suele integrar grupos con los mayores, o bien conforman en algunos casos sus propias agrupaciones. La porción de niños/as de cuatro a seis años de edad se ha encontrado, a pesar de su corta edad, en varias oportunidades en calle sin la compañía de adultos, pero sí acompañados regularmente por hermanos algunos años mayores (cuyas edades van de los 8 a los 12 años). Por último, son las franjas que van de los diez a los doce años de edad y la que corresponde a los niños/as de cero a tres años las que menos aparecen el circuito de calle. Estos últimos, cuando han sido registrados, siempre se han encontrado acompañados de adultos.

En relación a las actividades que realizan, la mayoría se refiere al cuidado de autos, realizado generalmente por adolescentes. Otra actividad es el pedido de monedas a los automovilistas en los semáforos; en menor medida los niños/as piden a peatones que circulan por el lugar o al interior de los comercios. La venta de artículos varios (trapos de piso, flores, biromes, llaveros, etc.) es menos común en la zona, pero se han registrado situaciones. Se desempeñan también en el lavado de autos (adolescentes en este caso) y abriendo puertas de taxis (esto último en las cercanías de los bancos y en horario matutino exclusivamente). Son poco frecuentes otras actividades de generación de ingresos realizadas por los niños/as, tales como actividades artísticas (malabarismo, por ejemplo).

Con respecto a la continuidad de las situaciones, hay niños/as y adolescentes que tienen una «parada» fija en la cual, además, respetan un horario determinado; hay otros puntos en los cuales es frecuente la presencia de distintos

\footnotetext{
${ }^{2}$ La ciudad de Rosario se encuentra dividida a nivel municipal en distritos administrativos descentralizados y es en función de los mismos que se organizan los equipos de trabajo.
} 
niños/as que ocasionalmente utilizan la parada alternando con otros; así también situaciones registradas en una sola oportunidad.

\section{La construcción de las miradas}

A continuación, se presentan los principales resultados de la investigación, ordenados a partir de los objetivos específicos y los ejes de análisis en los cuales estos fueron desglosados:

a) la representación de la calle como espacio, presentada a partir del análisis de las relaciones sociales establecidas en calle, los espacios construidos en el ámbito callejero y los modos de organización de las actividades.

b) la representación acerca del concepto de trabajo, a partir del análisis del impacto subjetivo generado en los niños/as por su participación en la manutención del grupo familiar, el uso y manejo del dinero, las «trayectorias» laborales del grupo familiar y los modos de nombrar las actividades de generación de ingresos que realizan en calle.

c) otras actividades diarias ajenas a la situación de calle y la distribución del tiempo en las actividades de la jornada.

d) actividades lúdicas llevadas a cabo en la cotidianeidad de la estadía en calle y las pausas o continuidades en el desarrollo de las actividades en calle.

\section{a) Representación acerca de la calle como espacio}

El desarrollo de este tema se presenta en función de tres ejes de análisis. El tratamiento del primero de ellos, las relaciones sociales establecidas en la calle, está fundamentado en la idea de que las modalidades que adquieran allí los vínculos, sumadas al uso y la apropiación o no de los espacios que este ámbito ofrece, marcarán las características de la estadía en la calle, aportando a la generación de una determinada mirada y sensación de lo que la misma significa para los niños/as y, por tanto, aportando a la construcción de su propia representación como sujetos, en tanto partícipes de situaciones cotidianas que en ella se desarrollan.

Siguiendo en esta línea argumental, y considerando que las relaciones sociales son generadoras de la construcción de la propia autorrepresentación, se considera que los elementos constituyentes de la misma, la identidad y la autopercepción, son establecidos en relación con un «otro» (Hernando, 2000); y, a su vez, dependen de la conjunción de una serie de factores relacionados con la historia de vida y las experiencias y relaciones sociales establecidas que imprimen un determinado modo de constitución de los sujetos como tales. Simultáneamente, tal como afirma De Lauretis (1996), es a partir de la autorrepresentación que el sujeto se manifiesta de forma pública, es decir, para los Otros, constituyéndose un proceso dialéctico.

Los niños/as concretos a los que se hace referencia en este trabajo suelen agruparse de diverso modo para las actividades que realizan, generando vínculos entre sí. Las modalidades que adquieren estas agrupaciones en la zona son mayoritariamente las llamadas por Lapenna y Lezcano (1995) «unidades domésticas entre pares» (UDEP), en las cuales los miembros pueden o no tener entre sí vínculos de consanguinidad, pero en las que no existe la figura de un adulto y las decisiones son tomadas horizontalmente. 
Dentro de esta categoría, se encuentran aquellos grupos en los que los vínculos son preestablecidos por la consanguinidad (hermanos, primos o tíos y sobrinos de una misma edad que llegan y se retiran en la mayoría de los casos en forma conjunta del espacio de la calle), como también aquellos construidos en el transcurso de la estadía en calle y retroalimentados en muchos casos a nivel barrial.

Es siempre común la referencia hacia un otro en el relato y un contacto aunque sea mínimo establecido con ese otro/s. Este reconocimiento aparece de diversas formas pero existe casi en la totalidad de los casos una referencia (barrial, de identificación de actividades, «cuida autos como yo», generacional, «tienen mi edad», o son «más grandes», etc.).

El hecho de estar solo en calle suele estar asociado a haber transcurrido mucho tiempo en la misma y, por lo general, se trata de adolescentes. Pero no están solos todo el tiempo ni todos los días, sino que esta situación alterna con estar acompañados por sus pares.

La modalidad denominada «unidades de base familiar nuclear» (UDBFN), conformada por niños y adultos que tienen vínculos biológicos de primer orden -padre, madre- (Lezcano, Lapenna, 1995), no tiene un peso significativo en la realidad de la zona. Por el contrario, se han registrado sólo algunas situaciones en las que un familiar mayor de edad estuviera acompañando al niño/a en la situación de calle. En las mismas, los adultos presentes no participan, por lo general, activamente en la tarea que realizan los niños/as, sino que juegan el rol de acompañantes.

Tampoco se ha notado una vinculación con otros adultos que realizan actividades comunes en calle, los cuales no establecen, salvo excepciones, vínculos con los niños/as.

Volviendo a la vinculación entre pares, ésta es realizada muchas veces a modo de «acompañamiento», según lo que puede observarse en terreno. En este sentido, es posible inferir que la estadía en calle proporciona un ámbito de construcción de relaciones sociales aún cuando no se esté realizando una determinada actividad de generación de ingresos económicos en el tiempo completo de la jornada. Construidos previamente, o producto de la estadía en calle, los vínculos forjados suelen plantearse en términos de amistad y es notorio cierto grado de solidaridad en relación al reparto de las actividades que realizan. En el caso de los «cuidadores de autos», la misma aparece a partir de la organización de los integrantes de los grupos, los cuales se «turnan» para dirigirse a los autos (sobre todo en los días y/u horarios de escaso movimiento en la zona, lo cual puede ser interpretado como modo de repartir los escasos ingresos potenciales entre todos con cierto grado de equidad).

Son configuradoras, además, de la estadía en calle las relaciones establecidas con quienes transitan la calle regularmente y/o viven o trabajan en la zona. En relación a este grupo, los vínculos que se conforman oscilan entre dos extremos: la agresión o violencia, por una lado, y la caridad y filantropía por el otro.

En el primero de los casos, y sin pretender caer en una mirada ingenua de los niños/as que los coloque en una posición de «inocencia» absoluta, es notoria la violentación generada hacia ellos en la manera de relacionarse; desde amenazas concretas de distinto tipo hasta referencias en relación a la «mo- 
lestia» que generan. Si bien estas situaciones no se producen con frecuencia, es llamativo lo explícito de las mismas en cuanto al nivel de violentación mencionado y en cuanto a la culpabilización de los niños/as por su situación que se deja entrever en algunos relatos. En estos casos, las reacciones de los niños pasan por el miedo, el rechazo y/o la agresión, de acuerdo a sus particularidades.

Desde la otra mirada, basada en el reforzamiento de prácticas caritativas, se parte de lo que Da Silva Siqueira (1993) denomina «un modo religioso y mesiánico de comprensión de la problemática», en la que el niño de la calle aparece en el discurso como un ser indefenso, víctima de su condición y en la que se generan ciertos comentarios donde se los ubica en este lugar y una serie de acciones en consecuencia (entrega de ropa, calzado, etc.).

Este tipo de situaciones dan cuenta del estigma que se genera en relación a la figura del «chico de la calle». El ser «niño de la calle» hace del mismo alguien a quien se debe rescatar, y/o rehabilitar, convirtiéndolo en un objeto social (Roze, et. alli., 1999).

Respecto de las relaciones que tienen lugar con los comercios de la zona en los cuales algunos de los niños tienen establecida su «parada», éstas son planteadas en términos de cordialidad, lo cual explica la larga permanencia de los mismos en esos lugares. Es decir, al establecer un espacio específico de trabajo continuo en el tiempo, existe siempre un acuerdo implícito o explícito con los dueños y/o empleados de los negocios aledaños.

La referencia al espacio utilizado por los niños/as para la realización de sus actividades nos introduce en el segundo de los ejes a analizar, los espacios construidos en el ámbito callejero, teniendo en cuenta que para muchos niños/as, la calle se constituye desde temprana edad como el ámbito de referencia para el acceso a la supervivencia diaria.

Pero, además, la calle como espacio público y espacio social, se constituye como espacio de luchas simbólicas cotidianas, formando una parte importante en el desarrollo de los habitus, definidos por Bourdieu (1994) como esquemas de percepción y apreciación de las prácticas sociales y condiciona la forma de actuar, pensar, sentir las categorías de percepción del mundo.

La calle ocupa también un papel de relevancia en la conformación de identidades, aún con mayor peso en los casos en que existe una marcada desafiliación comunitaria y en los que no hay espacios de contención familiares e institucionales. Pero las mismas no deben fijarse exclusivamente en este aspecto (niño/a sujeto a la calle). En este sentido, el concepto «en situación» permite marcar la diferencia respecto de la tradicional denominación «niño de la calle», en tanto hablar de un niño/a «de» la calle no permite pensar en sus identidades como dinámicas ni cambiantes ni tener en cuenta la existencia de múltiples pertenencias. Esto niega la posibilidad de otros procesos de desarrollo de su subjetividad, ligándola exclusivamente al soporte de la calle.

Dentro de estos otros soportes posibles, se encuentra el barrio, el cual aparece en el relato de los niños/as como un espacio que, lejos de ofrecer protección o sostén, es de mayor peligro que la calle. En este sentido, se ubican ciertas referencias realizadas en torno al consumo de drogas, o la existencia de enfrentamientos barriales con los cuales los niños/as no quieren involucrarse. 
La realidad de estos niños/as, paralelamente, marca un proceso contrario al que Merklen (2005) denomina como la inscripción territorial de los sectores populares producida, según el autor, a partir de la década del '80. Desde esta mirada, aparece el barrio como el ámbito de desarrollo de movimientos cooperativos, de movilización y de protesta pero, además, como el único lugar desde el cual se accede para, en términos del autor, «redondear» los ingresos familiares. En este sentido, la inscripción en estructuras de vecinazgo supone el acceso a los medios fundamentales para la supervivencia.

Ahora bien, en el caso de los niños/as en situación de calle, no se estaría produciendo este soporte barrial suficientemente fuerte para mantenerse dentro de los límites del barrio. De hecho, se torna necesario salir del mismo para obtener ingresos y recursos adecuados para la manutención del grupo familiar y esta salida es realizada en forma individual y/o familiar y no por medio de la movilización colectiva. Paralelamente, el barrio no ha podido ofrecer espacios de contención y/o de socialización necesarios, o bien éstos, por diversos motivos, no han podido ser aprovechados por estos niños/as y adolescentes. Por el contrario, aparece en estos casos como lugar donde, como se adelantaba anteriormente, ciertas cuestiones indeseables desde su perspectiva pueden permanecer ocultas. En contraposición, el espacio de las avenidas, centros comerciales o espacios verdes otorgan otra visibilidad a este tipo de situaciones.

Cabe preguntarse si se estaría produciendo un nuevo proceso de «territorialización» de la calle, consolidándose un «lugar»para estos grupos en tanto el aprovechamiento de este espacio marca la construcción de un sentido de pertenencia.

En este sentido, es en todos los casos clara la referencia, manifestada por los mismos sujetos y/o inferida a través del trabajo en terreno, de la permanencia en una misma «parada» o esquina específica a lo largo del tiempo. Asimismo, cuando estos espacios son compartidos con los pares, existe de forma general un acuerdo previo manifiesto.

Estas consideraciones permiten inferir modos de organización en las actividades cotidianas, de acuerdo a los sujetos concretos a los que se haga referencia. Por lo general, los adolescentes que realizan actividades en la zona se organizan en cuadras fijas, aunque las mismas son compartidas en función de los distintos horarios del día, lo cual da cuenta de cierto conocimiento recíproco y de convenios establecidos. En estos casos, cada integrante de los grupos conformados participa activamente en la tarea que se esté llevando a cabo.

Distinta es la modalidad que suele darse con aquellos niños/as que asisten a la parada o punto determinado junto a algún adulto familiar (madre 0 padre); en estos casos (numéricamente menores que los anteriores) aparece la mencionada figura del adulto «acompañando» a los niños/as. Esto que en primera instancia podría leerse como un «aprovechamiento» de los mismos, puede verse también desde una intención de protección frente a determinadas situaciones de peligro que pudieran surgir. Permanecer «ajena/o» a la situación permitiría así una observación más minuciosa del espacio completo donde el niño/a realiza la actividad (esta argumentación es dada en algunos casos por las mamás). 
Una modalidad que suele darse en estos casos es que la mamá vaya a la parada con uno o algunos de sus hijos más chicos, quienes se quedan cerca de ella bajo su protección, mientras los/as más grandes realizan alguna actividad. Cuando, a diferencia de esta situación, los hermanos van sin ningún adulto, es común también el acompañamiento de unos con otros en una misma esquina o en esquinas contiguas. Siempre se hace referencia al conocimiento de la ubicación del otro y en oportunidades se agrupan de a dos para acompañarse mutuamente.

En líneas generales, es posible afirmar que en la estadía en la calle está siempre presente la referencia hacia otro que acompaña o que participa activamente, y con el cual o los cuales en cualquiera de los casos se ejerce recíprocamente una suerte de identificación. Se produce, además, la reafirmación de un espacio propio, construido individual o colectivamente que, más allá de los beneficios económicos que puede permitir en términos del aprovechamiento de puntos estratégicos de gran afluencia de gente, da cuenta de la constitución de un sentido de pertenencia hacia ese lugar. Por otro lado, entre ellos, la permanencia en un espacio permite la asociación de un lugar con una persona o grupo.

\section{b) Representación acerca del concepto de trabajo}

Este segundo tema surge con el interés de indagar en las significaciones que adquiere el trabajo para estos niños/as y establecer si existe para ellos/as una vinculación entre éste y las actividades que realizan en calle. Es decir, se intenta caracterizar estas actividades e identificar si los niños/as se piensan a sí mismos como trabajadores/as. En primera instancia, a través de la indagación en el impacto subjetivo generado en los niños a raíz de su participación en la manutención del grupo familiar.

En líneas generales, y aún más en lo que respecta a los niños/as de menor edad, se deja notar en los discursos cierto grado de obligatoriedad para la obtención de dinero diario. Esta no aparece necesariamente cargada de sentimientos de angustia, sino que en ocasiones se percibe cierta naturalización de las actividades y el hecho de obtener más o menos dinero se torna una especie de «desafío» entre los niños/as.

En ocasiones, el sentido de la obligación se vincula, según puede inferirse del relato, con una responsabilidad creada por los padres y que se manifiesta en montos específicos que los niños/as deben obtener. En otros casos, aunque no frecuentes, esta responsabilidad creada en los niños/as adquiere rasgos de explotación, en tanto son involucradas amenazas o violencia física concreta hacia ellos por parte de los padres o adultos responsables. Otras veces, la voluntad de obtener dinero aparece presente en el relato en términos de necesidades familiares para gastos específicos, vinculados a necesidades concretas.

El grado de responsabilidad adquirido por los niños/as puede ser inferido, además, de ciertas actitudes, posturas y gestos desarrollados para la obtención de dinero, que se relacionan con modos de hacer adquiridos por el recorrido en calle.

La responsabilidad creada y muchas veces sentida por los niños/as también se manifiesta en el cuidado de los hermanos, introduciéndonos al tema 
del trabajo doméstico. Aquí, la cuestión adquiere una connotación de género al tratarse, en la mayoría de los casos, de niñas que se quedan en el hogar. En varias oportunidades, como se desprende de los relatos, los varones son «seleccionados» para el desarrollo de las actividades en calle, mientras que las niñas «ayudan» en las tareas domésticas, las cuales se caracterizan por una nota distintiva: la invisibilidad. Invisible en su perspectiva privada y en su perspectiva económica, ya que aleja al trabajo doméstico de su papel en el mercado. Tal como afirma Rotondi (2000), el trabajo doméstico no aparece como una actividad material y productiva, pese a constituirse como pilar de la producción y reproducción social.

Respecto de los adolescentes presentes en el circuito, no es tan clara la referencia a los motivos por los cuales realizan una determinada actividad en calle. Esto sale a la luz en la escasa cantidad de registros que pudieron ser agrupados en este eje de análisis respecto a niños mayores de catorce años de edad aproximadamente. En lo que respecta a este grupo, es posible afirmar que, en líneas generales, las pocas referencias realizadas apuntan a la colaboración en el hogar y al uso del dinero para gastos personales, ambos de forma simultánea. Esta enunciación nos lleva al segundo eje de trabajo planteado para este tema, el uso y manejo del dinero, a partir del cual se intenta buscar el grado de autonomía o heteronomía que poseen los niños/as respecto del dinero que obtienen mediante su trabajo.

Esta clasificación que realizan Lezcano y Lapenna (1995) tiene que ver con el manejo personal del dinero en el primer caso y la «colaboración» con el grupo familiar en el segundo. Tomando esta tipología para la población del presente estudio, las situaciones oscilan entre la heteronomía y una combinación de ésta con la autonomía relativa.

En el primer grupo se hace referencia a aquellos niños/as (la mayoría, numéricamente hablando) cuyos ingresos son exclusivamente manejados por adultos responsables de los mismos. Este tipo de administración del dinero aparece en el relato de los niños/as o bien en el mismo momento de la actividad en calle, al ser llevado el dinero obtenido al adulto presente.

En el segundo tipo de situaciones, los niños/as refieren cierto control personal del dinero que obtienen por su actividad, aunque el mismo no es absoluto sino que corresponde a una parte (para golosinas, visitas al cyber, etc.). Como se infiere, esta porción es destinada a gastos no relacionados con bienes de primera necesidad, tales como ropa, calzado o alimentos, sino que éstos quedan a cargo de la administración del dinero de los adultos.

La alta proporción, casi exclusiva, del uso y manejo del dinero por parte de los padres, deja entrever dos cuestiones fundamentales: la primera de ellas se relaciona con que, contrariamente a lo que suele aparecer en el imaginario social, estos chicos tienen un contacto permanente con sus familias, conviven con ellas.

El segundo aspecto que deja entrever el alto grado de heteronomía del manejo del dinero es la comprobación de la pertenencia de los niños/as a familias y comunidades de sectores sociales altamente vulnerables. En las mismas, es necesario el despliegue de diversas estrategias de supervivencia que implican la incorporación de todos o al menos la mayoría de sus miembros, al mercado de trabajo, generalmente, del mercado laboral informal. 
Esta consideración se relaciona con otro de los ejes indagados, las trayectorias laborales del grupo familiar. En relación a este tema, es posible afirmar que en el relato de los niños/as no existen referencias acerca de la existencia de algún trabajo estable en el contexto familiar. Por el contrario, es común la organización de actividades informales llevadas a cabo paralelamente por todo el grupo familiar.

La situación de calle, en este sentido, suele ser naturalizada como un medio más de subsistencia, lo cual permite hablar de procesos de exclusión permanentes que retroalimentan los caminos futuros de las trayectorias de los sujetos. Es decir, los mismos procesos de exclusión sufridos por los adultos van haciendo cada vez más dificultosa su inserción actual o futura a un mercado laboral estable, lo cual genera la reproducción de las actuales condiciones de existencia en las que sus grupos familiares se encuentran inmersos.

Estas características de los sujetos, entre las que Rotondi (2000) menciona la historia laboral (historias inestables, fuera del mercado formal, con mínimas o casi nulas posibilidades de reconversión laboral, ausencia de capacitación), plantean inserciones relacionales débiles que podrían acercarse a la desafiliación. Este proceso es notorio en el hecho de que los adultos desarroIlan en muchos casos las mismas actividades que sus hijos para lograr la subsistencia, lo cual marca la ausencia o la inadecuación de mecanismos educativos y/o de capacitación en la historia de los sujetos que le permitan la inserción en el mercado laboral.

La pregunta a hacer a continuación es: en todo este proceso en el que los niños/as se ven involucrados activamente, cómo se piensan en relación a las actividades que realizan. Este aspecto puede inferirse, entre otras cuestiones, a partir de la indagación en los modos de nombrar las actividades de generación de ingresos que realizan en calle. Esto está íntimamente vinculado a la definición de su autorrepresentación como trabajadores o no y en líneas más generales, a la significación y finalidad que esta actividad tiene para ellos/as.

La actividad que realizan aparece en el discurso en términos de trabajo, sólo en niños/as cuya edad supera los catorce años y no en todos los casos. Las menciones en este sentido se encuentran siempre relacionadas a una preocupación por la escasez de trabajo o a un notorio entusiasmo cuando se da la situación opuesta.

Estas referencias coinciden con el tipo de actividad de cuidado o lavado de autos, por lo que podría inferirse que la representación del trabajo está vinculada con actividades que generen algún tipo de producción o servicio que no se produce en el pedido de dinero en los cortes de semáforo, por ejemplo. En estos casos, hay también una referencia a cierta continuidad prolongada en el tiempo de actividades de este tipo en el ámbito callejero.

En el resto de las situaciones, los cuales corresponden a niños/as de corta edad, las referencias que se realizan varían entre la utilización de términos como «pedir», «ir», «hacer plata». Aquí, el encuadre de la actividad está relacionado con la mendicidad y fuertemente atravesado por determinadas estrategias que los niños/as van desarrollando para activar la caridad de los «clientes» potenciales. Se encuadra así en lo que da Silva (1993) denomina una relación ideológica en la que se recibe una «dádiva» a cambio del sentimiento reconfortante de un «cliente». 
Sin embargo, la correspondencia entre esta clase de afirmaciones y los tipos de actividad basados en la mendicidad no es absoluta, sino que en algunos de los casos está involucrada la venta de artículos varios, por lo que cabe interrogarse sobre qué aspectos provocan que un niño/a y/o adolescente se piense a sí mismo como un trabajador/a.

Algunas de las hipótesis ya fueron planteadas. De forma general, son los más grandes y con un largo recorrido en actividades de este tipo los que se piensan como tales. Por otra parte, la mendicidad o el popularmente llamado «mangueo» no es interpretado en ninguno de los casos como una actividad laboral, aunque implique el mismo gasto energético y la misma duración temporal que otro tipo de actividades. De todos modos, la venta de artículos tampoco es vista como trabajo por quienes la realizan, aunque vale aclarar que la misma es efectuada por niños/as con alguna trayectoria reciente en la mendicidad. Este hecho podría marcar la imposibilidad de un corte de una actividad con otra en su percepción.

c) Otras actividades diarias ajenas a la situación de calle, la distribución del tiempo en las actividades de la jornada

El fundamento de este objetivo se relaciona con el interés de mostrar qué otras identidades y pertenencias adquieren los niños/as más allá de la calle. Para esto, son planteados dos ejes de análisis. En el primero de ellos, definido en función de las actividades diarias ajenas a la situación de calle, cobra especial relevancia el concepto de niños/as «en situación» de calle, que reemplaza al tradicional niño «de la calle», en tanto permite recuperar, a través de la ubicación o no del niño/a en otros ámbitos, la dinamicidad de los procesos de construcción identitarios y movilizar sus soportes.

Los ámbitos de socialización son de gran importancia para la construcción de la autorrepresentación de los niños/as, en tanto su autopercepción se constituye a partir de una serie de factores que condicionan su comportamiento, como el ámbito familiar, la relación social, las experiencias de vida individuales, y las maneras como estos inciden en la construcción de su subjetividad (Green, 1981). En este sentido, son también de relevancia las participaciones de los niños/as en los ámbitos institucionales. Respecto de la misma, dicen Roze y Pratesi (1999) que la calidad y el tipo de instituciones a las que el niño se somete determinan su identidad y sus condiciones de existencia y son parte definitoria de su personalidad.

En el análisis de los registros en relación al tema de la inclusión de los niños/as en otras actividades ajenas a la situación de calle, adquieren centralidad dos espacios: la escuela y las actividades deportivas llevadas a cabo en clubes de la zona.

Respecto de la inclusión en el ámbito educativo, es posible afirmar que la referencia al mismo es realizada en un gran número de situaciones (siempre en escuelas cercanas a los domicilios). Así no estén asistiendo actualmente, es frecuente la referencia en el discurso a alguna institución a la que hayan asistido en el pasado.

Los casos de deserción escolar se relacionan casi de modo exclusivo con aquellos que se encuentran en edad adolescente. Sí es común la falta de correspondencia entre la edad de muchos de los niños y el grado o año al cual 
concurren, observándose un atraso escolar bastante marcado en la población infantil de referencia (denominado «sobre edad»).

Entre los más chicos, ha habido referencias concretas a maestros o a situaciones transcurridas en la escuela. Los más grandes, en cambio, no suelen dar detalles acerca del tema y las pocas referencias dadas se relacionan con problemas en el ámbito escolar que dificultan una real inserción educativa (relaciones violentas entre compañeros o problemas con los docentes por cuestiones disciplinarias); estas dificultades suelen relacionarse, a su vez, con problemas de orden económico.

Pero, como ya se mencionara, existen otras actividades que los niños/as alternan con la estadía en calle y que suelen relacionarse con lo deportivo/ recreativo. En otros casos, dedican tiempo de la jornada diaria a actividades en el barrio, en lugares cercanos al mismo o en casa de vecinos y/o familiares. En algunas ocasiones, el propio hogar es utilizado como lugar para el transcurso de parte de la jornada, lugar en el que se juega pero también en donde se participa o colabora en arreglos de la vivienda, por ejemplo.

En los casos en los cuales la situación de calle está instalada desde el propio espacio familiar, es más común encontrar situaciones en las cuales ésta imposibilita el fortalecimiento de otros espacios; esto es vivenciado en ocasiones con cierta angustia por parte de los niños/as, según aparece en sus relatos.

En relación a los horarios de estas actividades, es frecuente que se «acomoden» en relación a la situación de calle y viceversa. Es decir que, así como veíamos en párrafos anteriores que en ocasiones la situación de calle condiciona el resto de las actividades, otras veces éstas son modificadas en función de las mismas. Así, por ejemplo, el comienzo del año lectivo ha marcado algunas modificaciones en relación a los horarios en calle de los niños/as y adolescentes.

Pero más allá de las modificaciones producidas por estos aspectos, los niños/as suelen tener establecido un horario en el cual van a realizar sus actividades en calle. En algunas situaciones es fijo, lo cual suele vincularse con cierta organización de las actividades diarias cotidianas. El tiempo es repartido entonces en función de distintos espacios, tal como se planteaba con anterioridad. Este punto se relaciona con el eje referido a la distribución del tiempo en las actividades de la jornada.

La duración de la jornada en calle muchas veces se encuentra establecida en función de un horario fijo y prácticamente invariable. En otros casos, en consonancia con el establecimiento de ciertas pautas respecto del horario, aparece en el discurso cierta referencia a una especie de «turno» del día en el cual los niños/as se dirigen a la calle a realizar sus actividades, o bien a días específicos de la semana. Esta especie de «turno», de aproximadamente cuatro o cinco horas, permite la asistencia regular (en el caso de los que se encuentran escolarizados) a la escuela durante el período lectivo. Asimismo, el período de receso permite un mayor lapso de «tiempo libre» en el cual aumenta la frecuencia en calle.

En el caso de que estén en calle tanto de mañana como de tarde, generalmente al mediodía establecen un corte en el cual, según el relato, vuelven a sus hogares. En concordancia con esto, prácticamente no se han encontrado mu- 
chas situaciones de niños/as en calle en ese horario en ninguno de los meses del año que abarca el presente estudio. La llegada de la noche marca también un tope en el desarrollo de las actividades, alrededor de las 19 o 20 horas, según la época del año.

d) Actividades lúdicas llevadas a cabo en la cotidianeidad de la estadía en calle, las pausas o continuidades en el desarrollo de las actividades en calle

En este punto se intenta identificar aspectos lúdicos desarrollados por los niños/as en calle, así como la existencia y el uso de «tiempos libres» entre las actividades de generación de ingresos. Respecto al primero de los aspectos, las actividades lúdicas Ilevadas a cabo en la cotidianeidad de la estadía en calle, se indaga si el espacio de la calle, además de permitir la obtención de ingresos, es aprovechado también como ámbito de desarrollo de actividades lúdicas y, en caso de que así sea, qué características adquieren.

La aparición del juego en este espacio se considerará como una condición sumamente favorable para el desarrollo de estos niños/as, en tanto a través del mismo se fortalecen otros aspectos relativos a la creatividad, la resolución de problemas, el lenguaje, etc.

Es posible afirmar, a partir de la lectura y análisis de los registros correspondientes que, una vez más, las diferencias en la edad marcan diversos modos de involucramiento de los niños/as con las actividades lúdicas, al menos en las que se desarrollan en calle.

En niños de hasta doce años es común la alternancia de las actividades laborales con alguna clase de juego. Los mismos no tienen el soporte de algún tipo de elemento con el cual los niños/as interactúan, sino que es utilizado aquello que en el espacio de la calle haya podido ser conseguido. Sólo en algún caso son utilizados elementos traídos del hogar, pero de fácil traslado, como las «bolitas» $u$ otros juguetes pequeños.

En las actividades lúdicas es desplegada la creatividad y la imaginación para la adaptación a los recursos disponibles en ese momento. Las propuestas realizadas como operadoras de algún tipo de actividad recreativa (por ejemplo, de dibujo) fueron aceptadas instantáneamente y provocaron un corte en la actividad laboral de los niños/as.

Es notorio en todos los casos un gran interés por el aprovechamiento de los espacios de juego, o que permitan una pausa en las actividades cotidianas en calle. Esto puede explicarse, además de por la «natural» predisposición de los niños/as al juego, por la característica de los trabajos que llevan a cabo, rutinarios y monótonos.

Esta predisposición tiene su fundamentación, además, en lo que plantea Piaget (1986) cuando afirma que el juego, de imaginación o imitación, permite actuar sobre la realidad, transformándola y lograr con esa transformación satisfacer los deseos del que juega. Esta característica del juego, de suma importancia para cualquier niño/a, es sin lugar a dudas fundamental en la vida de estos niños/as en particular, que deben asumir responsabilidades a temprana edad. En relación con ello puede notarse que la aparición del juego se encuentra condicionada por el tiempo disponible para la obtención de dinero. En este sentido, es pasado a un segundo plano, efectuándose cuando se ha conseguido un mínimo de dinero, o haciéndose un corte en el mismo cuando ha transcu- 
rrido un rato considerado «demasiado largo» sin generar ingresos. Además, se dan casos en los que el mismo dinero obtenido es condición directa para la compra de ciertos juegos de interés, como en el caso de los cyber o video juegos. Son así los intersticios en la jornada o la culminación de la misma los que marcan los espacios posibles para la aparición de lo lúdico.

Por otro lado, el alto grado de creatividad desarrollado por los niños/as para jugar, puede estar vinculado a las carencias materiales a las cuales están habituados en otros aspectos de sus vidas cotidianas y que se trasladan a lo que tiene que ver con lo lúdico. En este sentido, dicen Roze y Pratesi (1999) que el juego es un sistema de comportamiento que extrae sus pautas de acción desde otros sistemas: la comida, el emparejamiento, el ataque agresivo y las ejecuta en otro contexto.

Por otra parte, las formas y características del juego dependerán de las historias singulares y los contextos de crianza de los niños/as. Estos, como sujetos singulares, son integrantes de grupos culturales con características propias cuyos valores, normas, costumbres y relatos ofician de referencia, marco y sostén y se organizan a través de particulares significados y creencias (Valiño, 2007). En relación a este aspecto, el relato de historias fantásticas contadas por los propios niños/as está también presente en las pausas de las actividades en calle. Por lo que puede inferirse, estas fábulas son transmitidas generacionalmente y provocan en los niños/as una especie de fascinación, permitiendo la actividad imaginativa y «desconectándolos» un rato de la actividad laboral.

Existe otro aspecto que adquiere relevancia en el análisis de las actividades lúdicas que llevan a cabo los niños/as en calle. Al ser planteado en un principio este eje, las situaciones que se venían dando marcaban una fuerte alternancia de las actividades laborales con el juego. La misma fue disminuyendo su intensidad en el transcurso de los últimos meses. Algunas de las hipótesis que explican esta modificación se relacionan con aspectos nombrados anteriormente en relación a otros ejes y/o en el momento de descripción de la población del estudio: en primer lugar, las edades de los niños en calle variaron a partir del comienzo del año lectivo, registrándose una mayor proporción de adolescentes (lo cual podría encontrarse relacionado con su deserción escolar). Tal como se dijo, son por lo general los más chicos los que llevan a cabo actividades relacionadas con el juego, en calle.

En segundo lugar, los períodos estacionales pueden haber influído en una disminución de las situaciones, ya que la estadía en calle se hace más dura y, por lo tanto, es de mayor urgencia la obtención de dinero rápidamente.

En tercer lugar, relacionado también con la época del año, ciertos espacios, como plazas, parques o fuentes de agua pierden su atractivo en época invernal. Como es notorio, es común el establecimiento de algún corte momentáneo en las actividades, lo que introduce al eje de las pausas y continuidades en las actividades en calle.

En general, es el ritmo del trabajo en calle el que marca los momentos en los cuales los niños juegan o socializan con sus pares u otros sujetos (como por ejemplo los cortes de semáforo para quienes están en las esquinas). Estos espacios son altamente aprovechados por los niños/as, ya sean habilitados por un externo como en el caso de la presencia del operador/a (algunas de 
las veces es solicitado explícitamente, lo que habla de la necesidad de un espacio de acercamiento, escucha y diálogo), ya sean generados por ellos/as mismos/as.

El común denominador en todos los casos es el acercamiento hacia un otro con el cual compartir experiencias y/o buscar algún tipo de acompañamiento. En este aspecto, juegan un papel fundamental los procesos de identificación con sus pares, con quienes establecen todo tipo de relaciones, por lo general cordiales y amigables, aunque con algunas excepciones de roces en función de la lucha por el espacio en calle que es, en definitiva, una lucha por el poder que pueden «manejar» desde sus realidades cotidianas.

\section{Reflexiones finales}

Aunque las imágenes que los niños/as tienen sobre sí mismos son diversas, es posible encontrar puntos en común: en primer lugar, no mantienen una afirmación homogénea de su autopercepción y sólo en pocos casos (de ciertos adolescentes) se mencionan a sí mismos como trabajadores. Por el contrario, las actividades que realizan se vinculan desde su percepción a un mandato familiar implícito o explícito y que obtiene condiciones óptimas para su reproducción, dada la precariedad de las condiciones de vida en sus contextos familiares.

Esta visión de sus actividades que aparece mayormente en términos de «pedir», «ir a la parada», «ir al semáforo» y otras expresiones similares se traduce en una percepción no laboral de sus prácticas y de sí mismos, y genera el desarrollo de tácticas concretizadas en gestos y actos específicos que activen en un otro actitudes caritativas.

Pero, simultáneamente, hay en muchos casos una manifiesta o implícita responsabilidad sentida por los niños/as en relación a la generación de ingresos económicos. Es decir, si bien no se desprenden de los relatos términos relacionados con el trabajo, la realización de la actividad no se ve alejada de cierto sentido de obligatoriedad.

La condición no laboral de estos niños/as se ve reforzada por el tipo de actividades realizadas, las cuales generalmente no se caracterizan por la producción o la comercialización de bienes, sino por no generar ningún «valor agregado» sobre algún bien o servicio.

No hay en los relatos referencia a algún proyecto laboral a corto o largo plazo. Es posible hipotetizar, en este sentido, una relación entre esta ausencia de imaginarios a futuro sobre posibilidades laborales y las historias laborales familiares relatadas por los niños/as, entre las que figuran el cirujeo, las changas de todo tipo y la repetición en el tiempo de las mismas actividades que los niños/as actualmente realizan. En ciertos casos, la misma situación de calle fue transitada durante la infancia o la adolescencia por otros miembros del grupo familiar, sean estos padres o hermanos mayores.

Es por todo esto que, sin querer caer en una visión simplista de la problemática de los niños/as en situación de calle, es posible pensar como un fuerte aspecto, o quizás como condición para su modificación, la generación de procesos masivos de inclusión social. 
En este sentido, y considerando que la exclusión, tal como plantea Castel (1991), se manifiesta a partir de un doble proceso de «desenganche»: con respecto al trabajo y con respecto a la inserción relacional, una política integral de atención y promoción de la infancia no puede pensarse de modo separado de la puesta en marcha de medidas que brinden a los adultos pertenecientes a los grupos familiares de estos niños/as, herramientas y posibilidades reales de inserción laboral, que trascienden a la política de infancia por sí sola.

A partir del establecimiento de una base en este sentido, se favorece la producción de un quiebre en el estado de cosas y adquiere viabilidad la desnaturalización de la situación por parte de estos grupos familiares y, por consiguiente, de los propios niños/as. En caso contrario, la misma se mantiene y reproduce como una estrategia más de supervivencia desplegada por las familias de los sectores populares.

En cuanto a las características generales de los niños/as, es posible establecer que se trata de una población heterogénea en cuanto a las distintas edades y frecuencias de la estadía en calle, pero de la que también se pueden extraer puntos en común. Se trata de grupos masculinos casi en su totalidad, de barrios cercanos a los lugares donde se «instalan» en calle, y a los cuales acceden sin la necesidad de un medio de transporte. Además, se trata de niños pertenecientes a familias numerosas, en las cuales ellos, en relación a los hermanos, han asumido ciertas responsabilidades familiares vinculadas a la obtención de ingresos económicos. Esta «selección» en ocasiones tiene que ver con ser el mayor entre los hermanos y también con ser varón. En este sentido, la mayoría de niños en calle tiene su paralelo en la gran cantidad de niñas que se hacen cargo de tareas del hogar o que ayudan en el cuidado de los hermanos más chicos. Esto no es cuestionado por los niños/as sino que, por el contrario, aparece en el relato fuertemente naturalizado.

Respecto a las relaciones establecidas con sus pares, es posible afirmar que, de modo general, hay un reconocimiento de los niños/as que se encuentran en calle en la zona entre sí. En los procesos de reconocimiento del otro es usual la búsqueda de una correspondencia de sus pares con lugares específicos donde estos desarrollan sus actividades, en una especie de reafirmación o reconfirmación de los espacios pertenecientes a cada uno. La profundidad de los vínculos aquí establecidos varía de acuerdo a las situaciones particulares, reforzándose en algunos casos a partir del ámbito barrial común. Pero los discursos sobre el par no son realizados en términos de hostilidad, sino que por lo general aparecen menciones de amistad, compañerismo o trato cordial entre ellos.

En la mayoría de los casos es notoria la necesidad de los niños/as de la creación de espacios de escucha por parte de un otro. El establecimiento de un diálogo no se ha tornado dificultoso en la generalidad de los casos sino que, por el contrario, ha sido posible generar, respetando siempre los tiempos de nuestros interlocutores, conversaciones fructíferas. En este sentido, los momentos de diálogo establecidos con los niños/as y logrados a partir de un proceso de acercamiento gradual, permitieron crear un ámbito donde contar experiencias, historias y, en el caso de los más chicos, desarrollar fábulas y relatos fantásticos.

Aparece un gran interrogante: ¿de qué manera explotar todo este potencial 
creativo, estas aptitudes y actitudes de los niños/as para que puedan crecer en esta etapa crucial del desarrollo humano? Esta pregunta obtiene un mayor nivel de complejidad si se tienen en cuenta las mencionadas condiciones de pobreza que atraviesan estas familias y las responsabilidades por los niños/as asumidas, pero sin duda deben ser el horizonte buscado desde el ejercicio profesional, en pos del respeto de los derechos de los niños/as y adolescentes.

En este sentido, las situaciones encontradas en el desarrollo del trabajo hablan de cierta correspondencia entre la mayor apropiación del espacio de la calle y la desvinculación de espacios institucionales (aunque una desvinculación total no ha sido la situación más encontrada). Este estado de cosas requiere de la creación y/o fortalecimiento de espacios viables para estos niños/ as que otorguen una alternativa real a la estadía en calle, cuando ésta ya se ha instalado como un territorio de pertenencia. Los procesos de territorialización se encuentran asentados en estos casos mayoritariamente en la calle. Esta característica no excluye la existencia de otros soportes subjetivos de estos niños/as, pero sí limita su construcción.

El espacio de la calle se constituye tanto como ámbito de obtención de dinero como de socialización y, a la vez, en algunos casos, como lugar de desarrollo de aspectos lúdicos. En este sentido, se encontraría de algún modo reemplazando (al menos para estos grupos de niños/as) a las funciones tradicionales cumplidas por los barrios. Esto no significa que categóricamente los niños/as hayan abandonado la participación en espacios barriales, pero sí que la necesidad económica que los impulsa a la calle estaría generando que sea allí también donde construyan muchos de sus vínculos con sus pares. Paralelamente, algunas referencias sobre los barrios destacan ciertos aspectos negativos de los mismos, donde cuestiones relacionadas a la violencia física y simbólica y al abuso de poder pueden pasar inadvertidas. Estas circunstancias, si bien no ausentes en el espacio de la calle, adquieren allí un mayor grado de visibilidad que permite acceder, paradójicamente, a una protección mayor de la que se hace posible en los barrios.

Con este tipo de consideraciones, no hay que ignorar que la situación de calle de niños y niñas implica una forma de vulneración de sus derechos y que es responsabilidad del Estado hacerse cargo de la misma. Entendiendo que es sólo desde la perspectiva universal de los derechos que puede englobarse a todos los niños/as y adolescentes dentro de la misma concepción de infancia, este trabajo intenta generar un aporte hacia el conocimiento de ese mundo infantil recortado en un tiempo y espacio específico pero, al mismo tiempo, atravesado por variables de nivel macro históricas, culturales, sociales, políticas y económicas que dan forma y determinan las condiciones de existencia de la infancia, o mejor dicho, de las infancias múltiples. 


\section{Bibliografía}

AMEZCÚA, M. El trabajo de campo etnográfico en salud: una aproximación a la observación participante. Granada, Index de Enfermería, 2000.

BOURDIEU, P. Las prácticas sociales. Buenos Aires, CEAL, 1994.

CASTEL, R. «La dinámica de los procesos de marginalización», en: Revista Topía. Año I, n. 2. Buenos Aires., 1991.

DA SILVA SIQUEIRA, J. «Algunas consideraciones sobre la cuestión de la crianza de los adolescentes de la calle», en: Servicio Social y Sociedad. Año XIV, $N^{\circ} 43$, San Pablo, Ed. Cortez, 1993.

DE LAURETIS, T. «La tecnología del género», en: Mora. № 2. Bs. As, Universidad Nacional de Buenos Aires, 1996.

GREEN, A. «Pasiones y destinos de las pasiones» en: Revista de psicoanálisis. T XXXVIII, n. 3, Asociación Psicoanálisis Argentina 1981.

GUBER, R. El salvaje metropolitano. Buenos Aires, Editorial Legasa, 1990.

HERNANDO, A. «La construcción de la subjetividad femenina» [en línea] Madrid: Instituto de Investigaciones Feministas, Universidad Complutense, 2000 [citado mayo de 2007]

Disponible en Internet en:

http:// www.razonypalabra.org

LAPENNA, M.; LEZCANO, A.»Condiciones de vida y estrategias laborales de los niños en situación de calle», en revista: Cátedra paralela, n. 1, Rosario,1995.

MAESTRE, J.La investigación en Antropología social. Madrid, Editorial Akal, 1990.

MERKLEN, D. Pobres ciudadanos. Las clases populares en la era democrática (Argentina, 1983-2003). Buenos Aires, Ediciones Gorla, 2005.

MORETA, P.; SERRA, F.; OJEDA, O. La investigación aplicada a la especificidad del Trabajo Social: elementos teóricos y operativos. Ficha de cátedra Práctica Pre- Profesional III, Lic. en Trabajo Social, Universidad Nacional de Rosario, 2004.

PIAGET, J. Estudios de psicología genética. Buenos Aires, Editorial Emecé, 1986.

ROTONDI, G. Pobreza y masculinidad: el urbano marginal. Buenos Aires, Editorial Espacio, 2000.

ROZE, J.P.; PRATESI, A.R. y otros. Trabajo, moral y disciplina en los chicos de la calle. Buenos Aires, Editorial Espacio, 1999.

VALIÑO, G. El juego en la infancia y en el nivel inicial. [en línea] Buenos Aires: Ministerio de Economía de la Nación, 2007 [citado mayo 2007]. Disponible en Internet:

http:// www.me.gov.ar 